Universidade Tecnológica Federal do Paraná - UTFPR

Campus Ponta Grossa - Paraná - Brasil

ISSN: $1981-3686 /$ v. 05, n. $02:$ p. $500-515,2011$

D.O.I.: $10.3895 / \mathrm{S} 1981-36862011000200002$
Revista Brasileira de Tecnologia

Agroindustrial

\title{
ELABORAÇÃO DE VINHO EM CULTURA MISTA DE LEVEDURAS NÃO- SACCHAROMYCES ISOLADAS DE JACA (Arthocarpus heterophyllus LAM) E Saccharomyces cerevisiae: AVALIAÇÕES QUÍMICA E SENSORIAL
}

\section{WINEMAKING IN MIXED CULTURE OF NON-SACCHAROMYCES JACKFRUIT'S YEAST (Arthocarpus heterophyllus LAM) AND Saccharomyces cerevisiae: CHEMICAL AND SENSORY EVALUATIONS}

\author{
Aline Bravo Barbosa Couto ${ }^{1}$; Poliana Deyse Gurak ${ }^{2}$; Claudete Corrêa de Jesus Chiappini ${ }^{3}$; Mauro Celso \\ Zanus $^{2}$; Gildo Almeida da Silva ${ }^{2}$; Selma Gomes Ferreira Leite ${ }^{1}$ \\ ${ }^{1}$ Instituto de Química, Universidade Federal do Rio de Janeiro - Brasil \\ alinebravo@ hotmail.com \\ ${ }^{2}$ Empresa Brasileira de Pesquisa Agropecuária - Embrapa Uva e Vinho, Bento Gonçalves - RS - Brasil \\ poligurak@ hotmail.com \\ ${ }^{3}$ Faculdade de Nutrição, Universidade Federal Fluminense - Niterói - RJ - Brasil \\ chiappini2@yahoo.com
}

\begin{abstract}
Resumo
Duas linhagens de leveduras não-Saccharomyces provenientes da microbiota da jaca - Pichia pijperi e Candida valida - foram inoculadas em associação com a linhagem de Saccharomyces cerevisiae em quatro diferentes processos de vinificação. A partir das análises químicas realizadas concluiu-se que a inoculação dessas leveduras não interferiu no processo de vinificação. Uma equipe de provadores treinados avaliou sensorialmente os vinhos através de Análise Descritiva Quantitativa e os resultados analisados por Análise de Componentes Principais e Análise de Variância. $O$ vinho inoculado com a linhagem de levedura Pichia pijperi apresentou atributos como odor indesejado e gosto indesejado, enquanto a linhagem Candida valida conferiu ao vinho atributos de aroma intensidade de aroma e intensidade de aroma frutado. Os resultados apontam o potencial de aplicação de leveduras provenientes da microbiota da jaca como culturas em vinificação.
\end{abstract}

Palavras-chave: vinificação; cultura mista; Arthocarpus heterophyllus Lam.; não-Saccharomyces; Pichia pijperi; Candida valida

\section{Introdução}

Ao longo da vinificação, a transformação do açúcar do mosto em álcool dá-se pela ação de leveduras e seu rendimento depende da velocidade do processo e, dentre outros fatores, da linhagem de levedura empregada. A função primária da levedura de vinificação - Saccharomyce cerevisiae é a de transformar os açúcares da uva em etanol, gás carbônico e outros compostos metabólicos sem desenvolver aromas desagradáveis (JOLLY et al., 2003b). Vinhos obtidos de um mesmo mosto e 
fermentados por leveduras distintas em condições idênticas podem apresentar diferenças devido aos diferentes produtos formados durante a fermentação (DA SILVA E DA SILVA, 1987).

A fermentação do mosto de uva em vinho é um complexo microbiano de reações envolvendo o desenvolvimento seqüencial de várias espécies de leveduras e bactérias (DA SILVA, 2003; COMBINA et al., 2005), podendo incluir fungos filamentosos (DA SILVA, 2003).

A qualidade do vinho está estreitamente relacionada com a ecologia microbiana da fermentação. Isso porque as leveduras metabolizam os constituintes da uva, principalmente açúcares, produzindo compostos voláteis e não-voláteis como produtos finais que influenciam no aroma e "flavor" característicos do vinho (ROMANO et al., 2003). Nos últimos cem anos atribuiuse maior importância às leveduras quando foi compreendida sua atuação na vinificação. Desde então foi possível um controle mais efetivo da fermentação e melhoria da qualidade dos produtos finais (JOLLY et al., 2003b).

Diferentes cultivares e safras de uva podem apresentar populações microbianas distintas, influenciando na quantidade e qualidade de produtos formados e comprometendo a padronização do produto final (DA SILVA, 2003).

Existe uma grande variedade de leveduras naturais - também chamadas de selvagens, indígenas ou autóctones - na superfície da baga das uvas (VINE et al., 1997). A principal fonte de leveduras indígenas no mosto são as uvas e os equipamentos usados para o seu processamento (PEYNAUD e DOMERCQ, 1959; ROSINI, 1984; LONVAUD-FUNEL, 1996). As leveduras nãoSaccharomyces são as espécies dominantes na uva (VAUGHAN-MARTINI e MARTINI, 1995; BOULTON et al., 1996; CONSTANTI et al., 1997) e durante o esmagamento, essas podem ser introduzidas no mosto (CUINIER et al., 1981; ROSINI, 1984.; HEARD e FLEET, 1988; AIZAC et al., 1991; JOLLY, AUGUSTYN e PRETORIUS, 2003a).

A dominância de leveduras e bactérias indesejadas pode vir a causar um "flavor" desagradável ao vinho - como o de sulfetos e mercaptanos - (DA SILVA E DA SILVA, 1987), fermentações interrompidas e vinhos defeituosos (GAFNER et al., 2000).

A possibilidade de manipulação de microrganismos no mosto de uva a ser fermentado não deve se restringir à população microbiana da uva. Phaff e Stamer (1987) demonstram que o solo, as plantas e, particularmente, os frutos são "habitats" de leveduras na natureza. Frutos possuem em sua microbiota leveduras selvagens devido à elevada concentração de açúcares simples, baixo pH e intensa visitação de vetores. Poucos estudos têm sido realizados sobre leveduras de frutos tropicais e, em geral, os resultados coincidem com os dados de áreas temperadas, revelando, entretanto, a presença de biótipos únicos.

Vários trabalhos apresentam a gama de possibilidades de espécies leveduras isoladas de plantas encontradas em praias e restingas do Sudeste brasileiro, com Candida sonorensis, 
Clavispora opuntiae, Pichia barkeri e Pichia cactophila. Plantas do Nordeste brasileiro, como caju, cajá, umbu e manga, apresentaram grande biodiversidade de leveduras, como Candida, Cryptococcus, Haltermannia, Rhodotorula, Sporobolomyces, Tremella, Hanseniaspora, Kloeckera e Pichia (MORAIS et al., 1995a; MORAIS et al., 1995b; ROSA et al., 1995; SANTOS et al., 1996; ABRANCHES et al., 2000).

Os primeiros estudos sobre leveduras não-Saccharomyces no vinho as fazem referência como organismos causadores de defeitos (AMERINE e CRUESS,1960; VAN ZYL e DU PLESSIS, 1961; RANKINE, 1972; LE ROUX et al., 1973). Porém trabalhos mais recentes, também indicam que o efeito de leveduras não-Saccharomyces na fermentação pode ser o principal aspecto de qualidade do vinho correntemente estudado em diferentes países com tradição enológica (GROSSMANN et al., 1996; JOLLY et al., 2003b).

Os potenciais benefícios e defeitos causados por leveduras não-Saccharomyces devem ser explorados visto que são as responsáveis pela maior influência na qualidade do vinho. Portanto deve-se conhecer sua importância na uva e no mosto - quais linhagens de levedura nãoSaccharomyces podem sobreviver e crescer - e os efeitos das práticas de vinificação sobre as mesmas (JOLLY et al., 2003c).

O conhecimento desses benefícios e defeitos indica a possibilidade de no futuro os vinicultores preferirem usar misturas de linhagens de Saccharomyces cerevisiae e nãoSaccharomyces como espécies de culturas "starter" (JOLLY et al., 2003c).

A vinificação em cultura mista, com leveduras não-Saccharomyces isoladas de diferentes frutas, pode resultar em vinhos com características de qualidade singulares. $\mathrm{O}$ isolamento de leveduras de frutas de clima tropical pode representar uma interessante proposta no desenvolvimento de vinhos específicos desse clima.

O presente trabalho tem por objetivo investigar a influência de leveduras nãoSaccharomyces isoladas de jaca, fruta típica de clima tropical, na qualidade química e sensorial de vinhos elaborados a partir de cultivar Cabernet Sauvignon.

\section{Material e métodos}

\section{Leveduras}

Empregou-se uma linhagem de Saccharomyces cerevisiae EMBRAPA1vvt97 proveniente da coleção do Laboratório de Microbiologia do Centro Nacional de Pesquisa da Uva e do Vinho (CNPUV) - EMBRAPA, e as linhagens de leveduras não-Saccharomyces, Candida valida e Pichia pijperi - isoladas de jaca (Artocarpus heterophylus Lam) - caracterizadas e identificadas no 
Laboratório de Biodiversidade de Leveduras, do Departamento de Microbiologia Geral, do Instituto de Microbiologia Professor Paulo de Góes - Rio de Janeiro, RJ (CHIAPPINI, 2004).

As linhagens não-Saccharomyces encontravam-se em meio de manutenção ágar GYMP contendo 0,5\% de Bacto ${ }^{\mathrm{TM}}$ Yeast Extract (Difco), 2\% de extrato de malte, 2\% de glicose, 0,2\% de fosfato de sódio monobásico e $2 \%$ de ágar a $25 \pm 1{ }^{\circ} \mathrm{C}$ e estocadas em óleo mineral estéril a $8 \pm 3^{\circ} \mathrm{C}$ (MORAIS et al., 1995; SANTOS et al., 1996).

\section{Vinificação}

Os tratamentos consistiram em empregar duas linhagens não-Saccharomyces - Candida valida e Pichia pijperi, codificadas como Pm e Pp, respectivamente, na vinificação em tinto em combinação com agente principal de fermentação a linhagem Saccharomyces cerevisiae Embrapa 1vvt. Os tratamentos estão apresentados na Tabela 1.

Tabela 1 - Tipos de vinhos elaborados com as diferentes leveduras Saccharomyces e não-Saccharomyces

\begin{tabular}{lcccc}
\hline & Vinho 1 & Vinho 2 & Vinho 3 & Vinho 4 \\
Tipo de levedura utilizada & $1 \mathrm{vvt}$ & $1 \mathrm{vvt}+\mathrm{Pp}$ & $1 \mathrm{vvt}+\mathrm{Pm}$ & $1 \mathrm{vvt}+\mathrm{Pp}+\mathrm{Pm}$ \\
\hline Legenda: & & \\
1vvt - levedura Saccharomyces cerevisiae & (vinho controle) \\
1vvt + Pp - levedura Saccharomyces cerevisiae & em associação com Pichia pijperi \\
1vvt+Pm - levedura Saccharomyces cerevisiae em associação com Candida valida \\
1vvt+Pp+Pm - Associação das três leveduras Saccharomyces cerevisiae, Pichia pijperi e Candida valida
\end{tabular}

As leveduras foram adicionadas ao mosto na combinação descrita na Tabela, tendo o tratamento 1 como testemunha. Depois de adaptadas a $500 \mathrm{~mL}$ de mosto $\left(30 \mathrm{~min}\right.$ à $25 \pm 1{ }^{\circ} \mathrm{C}$ ), as leveduras foram transferidas para o mosto a ser vinificado em quantidades suficiente para que cada linhagem fosse inoculada na concentração de $10^{6}$ células $/ \mathrm{mL}$ de mosto. $\mathrm{O}$ experimento seguiu um delineamento inteiramento casualizado com três repetições.

No processo de vinificação foi empregado o cultivar de uva Cabernet Sauvignon plantado em sistema de espaldeira e colhido por meio de colheita manual no mês de abril. As uvas procederam da região de Campo de Cima da Serra, Vacaria (RS) e, após a colheita, foram diretamente transportadas para a EMBRAPA-CNPUV para o processamento.

A vinificação foi realizada no Laboratório de Microvinificação da EMBRAPA Uva e Vinho. As uvas foram desengaçadas e esmagadas em desengaçadeira-esmagadeira (Pillan, Itália). O mosto obtido foi submetido à adição de metabissulfito de potássio de modo a fornecer $70 \mathrm{mg} / \mathrm{L}$ de $\mathrm{SO}_{2}$ livre. O mosto foi transferido para frascos (Pyrex) adicionados das leveduras nas combinações descritas (1vvt+Pp, 1vvt+Pm, 1vvt+Pp+Pm e 1vvt - testemunha) fechados com válvulas de Müller e acondicionados em câmara à 24C. A fase tumultuosa da fermentação teve 
duração de seis dias. Durante esta fase foram realizadas duas remontagens diárias, sendo realizada ao final desta fase a descuba. Ao longo da fermentação lenta foram realizadas três trasfegas - a primeira 24horas após a descuba, a segunda 18 dias após o esmagamento e a terceira 21 dias após a segunda trasfega. A fermentação malolática foi espontânea, sem inoculação de bactéria e teve início aos 30 dias após o término da fermentação tumultuosa e foi acompanhada por cromatografia em papel até ser verificada a ausência do ácido málico. Ao final da fermentação, os vinhos foram adicionados de metabissulfito de potássio de modo a perfazer $60 \mathrm{mg}$ de $\mathrm{SO}_{2}$ livre/L e transferidos para uma câmara climatizada a temperatura em torno de $0^{0} \mathrm{C}$ durante 15 dias para a estabilização. Após este período, os vinhos foram analisados quanto ao teor de $\mathrm{SO}_{2}$ livre, corrigidos para o teor mínimo de 50mg/L de $\mathrm{SO}_{2}$ livre quando necessário, trasfegados e envazados em garrafas de $750 \mathrm{~mL}$.

\section{Análises químicas}

O mosto da uva foi analisado em relação a pH, ${ }^{0} \mathrm{Babo},{ }^{0} \mathrm{Brix}$, acidez total e açúcares redutores totais em glicose (ART), segundo Riberéau-Gayon et al. (1976).

Os vinhos foram analisados quimicamente por meio das seguintes determinações: teor alcoólico - \%v/v, acidez total - mEq/L, acidez volátil - mEq/L, acidez fixa - mEq/L, $\mathrm{pH}$, açúcares redutores em glicose $(A R T)-g / L$, extrato seco $-\mathrm{g} / \mathrm{L}$, extrato seco reduzido $-\mathrm{g} / \mathrm{L}$, cinzas $-\mathrm{g} / \mathrm{L}$, alcalinidade das cinzas $-\mathrm{g} / \mathrm{L}, \mathrm{SO}_{2}$ livre $-\mathrm{mg} / \mathrm{L}, \mathrm{SO}_{2}$ total $-\mathrm{mg} / \mathrm{L}$, relação álcool em peso/extrato seco reduzido, taninos -g/L, polifenóis - I280, antocianinas - mg/L, intensidade de cor, I420\%, I520\%, I620\% (Ribéreau-Gayon et al., 1976) e Folin-Ciocalteau - mg/L de ácido gálico (Slinkard e Singleton, 1977; Waterhouse, 2005). Para a análise de Folin-Ciocalteau foi realizada uma curvapadrão.

\section{Avaliação sensorial}

Os vinhos submetidos aos quatro tratamentos - 1vvt, $1 v v t+P p, 1 v v t+P m$ e $1 v v t+P p+P m-$ foram avaliados sensorialmente para verificar se a aplicação das leveduras não-Saccharomyces influenciaram nos atributos sensoriais dos vinhos.

Uma equipe constituída por membros treinados "experts" avaliou os vinhos utilizando a Análise Descritiva no Laboratório de Análise Sensorial do Centro Nacional de Pesquisa da Uva e do Vinho (CNPUV) - EMBRAPA. A equipe foi composta por nove provadores treinados, sendo funcionários e estagiários da instituição, com faixa etária bem abrangente - de 19 a 64 anos de 
idade, formação profissional afim - Engenheiros Agrônomos, Técnicos em Agropecuária, estudantes de Enologia e Enólogos - e com no mínimo um ano de experiência em degustação.

A aplicação dos testes decorreu em cabine individual com apresentação de uma amostra de cada vez e foram oferecidos água e biscoito água e sal para a limpeza do palato entre as amostras.

Cada amostra foi avaliada quanto à intensidade percebida das características visual, olfativa, paladar e qualidade geral em ficha descritiva para vinhos tintos.

\section{Tratamento estatístico}

As análises químicas tiveram seus resultados tratados através da Análise de variância (ANOVA) e teste de médias de Tukey apresentando os pares de amostras que apresentaram diferença significativa ao nível de $5 \%$ de probabilidade $(\mathrm{p} \leq 0,05)$.

Os resultados da Análise Descritiva realizada na EMBRAPA-CNPUV foram analisados estatisticamente pela Análise de Variância (ANOVA) e Análise de Componentes Principais, utilizando o programa computacional Statistica $^{\mathrm{TM}}$.

\section{Resultados e Discussão}

\section{Avaliação química do mosto e do vinho}

O mosto inicial foi analisado logo após sua obtenção e apresentou valor de $\mathrm{pH}$ de 3,44, $20^{0}$ Babo, $21,5^{0}$ Brix e $212,5 \mathrm{~g} / \mathrm{L}$ de açúcares redutores totais.

Os resultados das análises químicas finais dos parâmetros etanol, acidez total, acidez volátil, acidez fixa, $\mathrm{pH}$, açúcares redutores totais - ART, extrato seco, extrato seco reduzido, cinzas, alcalinidade das cinzas, $\mathrm{SO}_{2}$ livre, $\mathrm{SO}_{2}$ total, intensidade de cor, $\mathrm{I} 420 \%$, $\mathrm{I} 520 \%$, $\mathrm{I} 620 \%$, taninos, antocianinas, "hue”,polifenóis e Folin-Ciocalteu são apresentados na Tabela 2.

O tratamento estatístico demonstrou não haver diferença significativa ao nível de $5 \%$ de probabilidade ( $\mathrm{p}>0,05)$ nas análises de densidade, álcool, acidez total, acidez volátil e acidez fixa ao final do processo de vinificação. Tais resultados indicam que a aplicação das leveduras nãoSaccharomyces Pichia pijperi $(\mathrm{Pp})$ e Candida valida $(\mathrm{Pm})$ não prejudicam a atuação da levedura de vinificação - 1vvt - na produção de etanol e não interfere no equilíbrio dos ácidos gerados ao longo da vinificação.

A análise de açúcares redutores totais também não apresentou diferença significativa ao nível de significância de $5 \%$ de probabilidade $(\mathrm{p}>0,05)$. 
Tabela 2. Valores médios e desvios-padrão (DP) das análises químicas realizadas nos vinhos

*Amostras que apresentaram diferença significativa ao nível de $5 \%$ de probabilidade $(\mathrm{p} \leq 0,05)$

\begin{tabular}{|c|c|c|c|c|}
\hline \multirow[t]{2}{*}{ Análises } & $1 v v t$ & $1 v v t+P p$ & $1 v v t+P m$ & $1 v v t+P p+P m$ \\
\hline & \multicolumn{4}{|c|}{ Média DP } \\
\hline Etanol $(\% \mathrm{v} / \mathrm{v})$ & $12,3 \pm 0,2$ & $12,2 \pm 0,5$ & $12,4 \pm 0,2$ & $12,3 \pm 0,2$ \\
\hline Acidez total $(\mathrm{mEq} / \mathrm{L})$ & $89,3 \pm 3,1$ & $92,7 \pm 6,4$ & $94 \pm 5,3$ & $98 \pm 6,0$ \\
\hline Acidez volátil (mEq/L) & $4,00 \pm 0,00$ & $4,33 \pm 0,58$ & $4,33 \pm 0,58$ & $4,33 \pm 0,58$ \\
\hline Acidez fixa $(\mathrm{mEq} / \mathrm{L})$ & $85,33 \pm 3,06$ & $88,33 \pm 5,86$ & $89,67 \pm 5,86$ & $93,67 \pm 6,03$ \\
\hline $\mathrm{pH}$ & $3,40 \pm 0,04$ & $3,40 \pm 0,061$ & $3,36 \pm 0,03$ & $3,33 \pm 0,08$ \\
\hline ART (g/L) & $3,23 \pm 0,12$ & $3,68 \pm 0,93$ & $3,50 \pm 0,72$ & $4,26 \pm 0,08$ \\
\hline Extrato seco $(\mathrm{g} / \mathrm{L})$ & $25,81 \pm 0,83 *$ & $27,75 \pm 0,22 *$ & $26,88 \pm 0,97$ & $27,47 \pm 0,65$ \\
\hline Extrato seco reduzido (g/L) & $22,82 \pm 0,69 *$ & $24,07 \pm 1,04^{*}$ & $23,38 \pm 0,97$ & $27,47 \pm 0,65^{*}$ \\
\hline $\begin{array}{l}\text { Relação álcool /extrato seco } \\
\text { reduzido }\end{array}$ & $0,540 \pm 0,017 *$ & $0,508 \pm 0,034^{*}$ & $0,533 \pm 0,023$ & $0,449 \pm 0,012$ \\
\hline Cinzas $(\mathrm{g} / \mathrm{L})$ & $2,08 \pm 0,15$ & $2,08 \pm 0,21$ & $1,90 \pm 0,05$ & $1,97 \pm 0,15$ \\
\hline $\begin{array}{l}\text { Alcalinidade das cinzas } \\
(\mathrm{mEq} / \mathrm{L})\end{array}$ & $24,5 \pm 2,18$ & $25,17 \pm 3,33$ & $23,67 \pm 0,58$ & $23,50 \pm 0,87$ \\
\hline $\mathrm{SO}_{2}$ livre $(\mathrm{mg} / \mathrm{L})$ & $54,06 \pm 1,96$ & $51,67 \pm 4,60$ & $60,21 \pm 4,80$ & $59,52 \pm 6,79$ \\
\hline $\mathrm{SO}_{2}$ total $(\mathrm{mg} / \mathrm{L})$ & $68,81 \pm 5,27$ & $79,81 \pm 22,82$ & $81,87 \pm 1,95$ & $83,96 \pm 7,69$ \\
\hline Intensidade de cor & $2,26 \pm 0,0534$ & $2,11 \pm 0,2609$ & $2,19 \pm 0,2260$ & $2,26 \pm 0,1692$ \\
\hline $\mathrm{I} 420 \%$ & $30,39 \pm 0,37$ & $31,15 \pm 1,07$ & $31,03 \pm 0,85$ & $30,70 \pm 0,68$ \\
\hline $\mathrm{I} 520 \%$ & $60,11 \pm 0,61$ & $59,13 \pm 1,74$ & $59,63 \pm 0,95$ & $59,95 \pm 1,09$ \\
\hline $\mathrm{I} 620 \%$ & $9,46 \pm 0,26$ & $9,74 \pm 0,75$ & $9,33 \pm 0,31$ & $9,34 \pm 0,45$ \\
\hline Hue $(420 / 520)$ & $0,51 \pm 0,015$ & $0,52 \pm 0,031$ & $0,52 \pm 0,021$ & $0,51 \pm 0,026$ \\
\hline Taninos $(\mathrm{g} / \mathrm{L})$ & $4,21 \pm 1,763$ & $5,48 \pm 0,503$ & $4,80 \pm 0,780$ & $5,39 \pm 0,801$ \\
\hline Antocianinas (mg/L) & $\begin{array}{c}640,24 \pm \\
45,910\end{array}$ & $\begin{array}{c}595,64 \pm \\
61,294\end{array}$ & $\begin{array}{c}488,57 \pm \\
98,588\end{array}$ & $559,86 \pm 123,695$ \\
\hline Polifenóis (I280) & $61,6 \pm 0,5$ & $61,9 \pm 1,7$ & $62,5 \pm 1,7$ & $63,2 \pm 0,9$ \\
\hline $\begin{array}{l}\text { Folin Ciocalteu (mg de ácido } \\
\text { gálico / L) }\end{array}$ & $2229,02 \pm 5,7$ & $2376,0 \pm 355,4$ & $2501,2 \pm 263$ & $3614,9 \pm 1694,9$ \\
\hline $\mathrm{DPPH}$ (mM de trolox/mg) & $9649,94 \pm 153$ & $\begin{array}{l}9476,12 \pm \\
507,8\end{array}$ & $\begin{array}{l}9799,61 \pm \\
607,7\end{array}$ & $10343,48 \pm 404,9$ \\
\hline
\end{tabular}

*Amostras que apresentaram diferença significativa ao nível de $5 \%$ de probabilidade $(\mathrm{p} \leq 0,05)$

Diferenças significativas foram encontradas nas análises de extrato seco, extrato seco reduzido e relação álcool em peso / extrato seco reduzido ao nível de $5 \%$ de probabilidade (ANOVA). O Teste de Tukey mostro que os tratamentos 1vvt e 1vvt+Pp apresentaram diferenças nas três análises acima e o tratamento $1 \mathrm{vvt}+\mathrm{Pp}+\mathrm{Pm}$ se diferenciou dos demais tratamentos na análise de extrato seco. Houve diferença também na relação álcool em peso / extrato seco reduzido entre os tratamentos $1 v v t$ e $1 v v t+P p+P m$ e $1 v v t+P p$ e $1 v v t+P p+P m$.

Essas diferenças encontradas nas análises de extrato seco, extrato seco reduzido e relação álcool em peso / extrato seco reduzido podem estar relacionadas: a um maior crescimento de células da levedura Pichia pijperi (Pp) que apresentou a maior taxa específica de crescimento das três linhagens e a inoculação de um maior número de células no tratamento $1 \mathrm{vvt}+\mathrm{Pp}+\mathrm{Pm}$ que foi acrescida das três linhagens de levedura. O possível aumento da biomassa final pode ter como consequiência uma maior liberação de subprodutos para o vinho aumentando o extrato seco. 
Os valores de $\mathrm{pH}$ para todos os tratamentos encontram-se dentro da faixa de 3,3 a 3,4 ideal para o desenvolvimento de aroma frutal mais intenso (VINE et al., 1997).

Diferenças significativas ao nível de $5 \%$ de probabilidade $(\mathrm{p}>0,05)$ não foram encontradas nas análises de cinzas, alcalinidade das cinzas, $\mathrm{SO}_{2}$ livre e $\mathrm{SO}_{2}$ total.

As análises I420\%, I520\% e I620\% representam o percentual das cores encontradas no vinho, significando respectivamente o percentual de cor laranja, de cor vermelha e de cor azul. O somatório dos três índices representa a quantidade de cor total do vinho e é expressa pela intensidade de cor (RIBÉREAU-GAYON et al., 2002). Essas análises não apresentaram diferenças significativas ao nível de significância de $5 \%$ de probabilidade $(\mathrm{p}>0,05)$. Isto indica que a aplicação dessas leveduras não-Saccharomyces não influenciaram na cor dos vinhos.

Os valores encontrados para intensidade de cor apresentam-se mais próximo do limite superior da faixa para intensidade citado por Ribéreau-Gayon et al. (2002) - absorbância de 0,3 a 2,8. Esse resultado indica a boa intensidade de cor dos vinhos elaborados.

Em geral os vinhos apresentaram, aproximadamente, a seguinte distribuição dos componentes de cor: $30 \%$ de amarelo, $60 \%$ de vermelho e $9 \%$ de azul.

Os valores de taninos, antocianinas e "hue" não apresentaram diferenças significativas ao nível de $5 \%$ de probabilidade $(\mathrm{p}>0,05)$. Grandes quantidades de taninos foram obtidas já que os valores encontram-se acima da quantidade máxima de 3,0g/L relatada por Ribéreau-Gayon e Peynaud (1958). Como os vinhos analisados eram recém-elaborados, sem terem passado pela etapa de maturação em barrica de carvalho e envelhecimento na garrafa, os valores de "hue" encontramse próximos de limite inferior da faixa para "hue" - 0,5 a 0,7 (RIBÉREAU-GAYON et al., 2002). Isso indica uma menor intensidade da tonalidade laranja, típico de vinhos jovens.

As análises de polifenóis e Folin-Ciocalteu não apresentaram diferenças significativas ao nível de significância de 5\% de probabilidade $(\mathrm{p}>0,05)$.

\section{Avaliação sensorial}

Os dados obtidos na Análise Descritiva com a equipe de provadores treinados realizada na EMBRAPA-CNPUV foram tratados pela Análise de Componentes Principais e pela Análise de Variância (ANOVA), ao nível de significância de 5\% de probabilidade.

A realização prévia dos gráficos "star-plots" possibilita a visualização do perfil de cada um dos tratamentos realizados, de acordo com os valores de intensidade fornecidos pelos provadores, sem a aplicação do tratamento estatístico. Cada atributo é representado por um eixo no gráfico "star-plots" e seus valores são comparados pelo distanciamento do ponto central, de convergência de todos os eixos. Os menores valores são os que mais se aproximam do ponto central e os maiores 
valores são os mais distantes. Os atributos foram distribuídos em dois gráficos, o primeiro apresentando os atributos intensidade de cor, de aroma, de sabor, de aroma vegetal, de aroma e de aroma frutado - Figura 1 - e o segundo apresentando os atributos odor indesejado, acidez, taninos, adstringência, amargo, gosto indesejado, persistência e doçura - Figura 2.

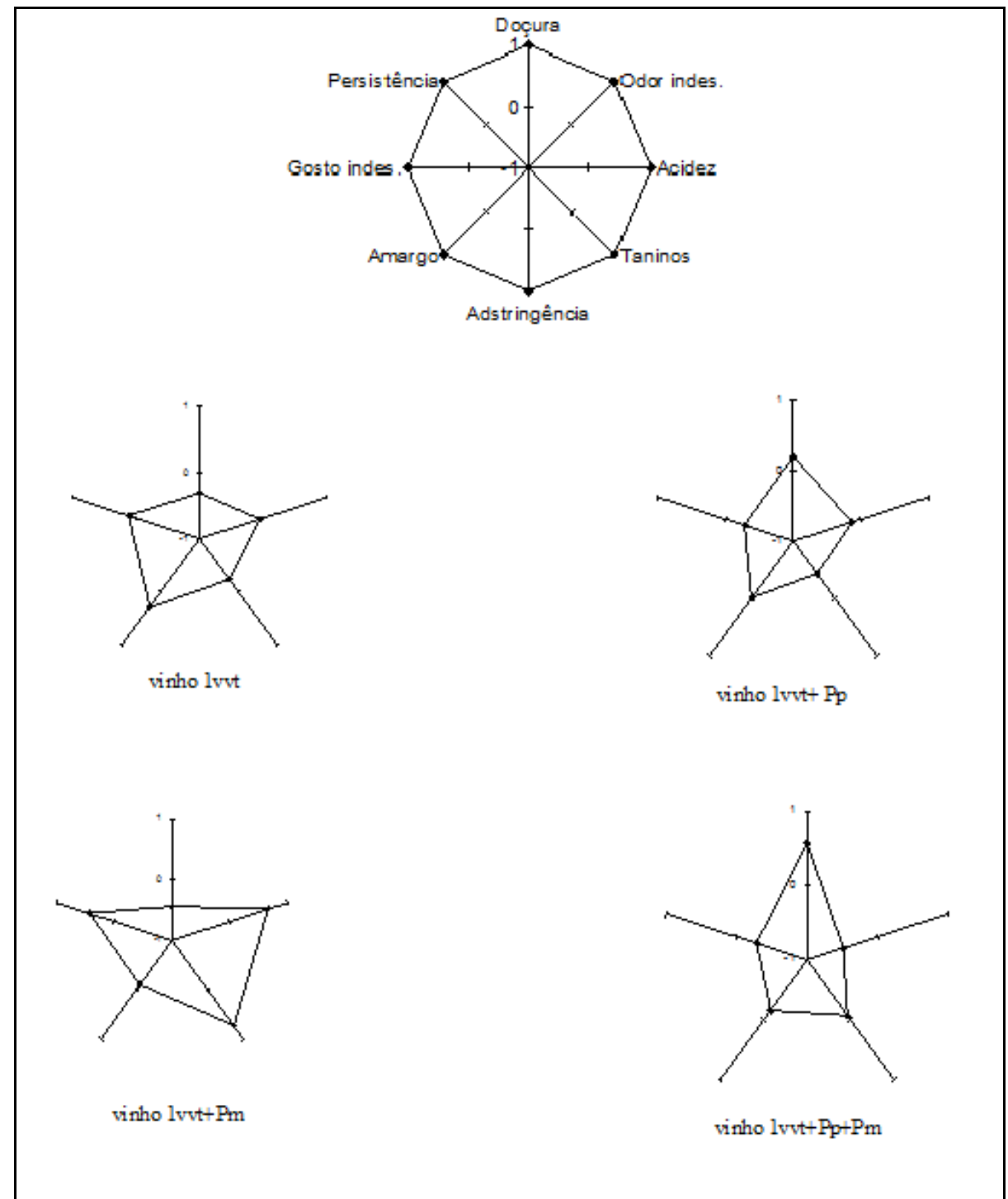

Figura 1 - Gráficos "star-plots" para os atributos de intensidade de cor, aroma, sabor, vegetal e frutado, indicando o perfil de cada tratamento

Conforme as configurações mostradas nas Figuras 1 e 2, o vinho 1vvt caracteriza-se por maior intensidade de aroma vegetal e menor intensidade de cor, doçura, odor indesejado e acidez. $\mathrm{O}$ aroma vegetal é um aroma varietal típico do cultivar Cabernet Sauvignon (VINE et al., 1997). Por ter apresentado maior intensidade deste aroma, os provadores consideraram o vinho testemunha 1vvt - rico em aroma proveniente da própria uva. Isto indica que os aromas secundários - obtidos pela ação de leveduras - não foram os predominantes neste vinho. 


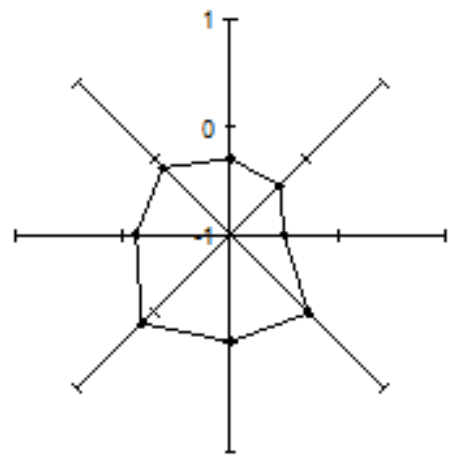

vinho lvvt

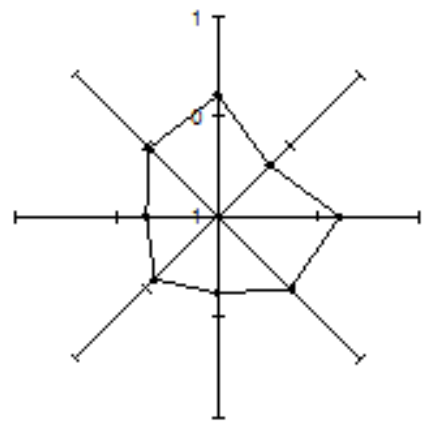

vinho lvvt+Pm

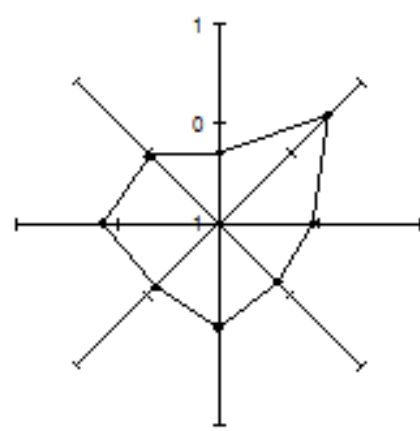

vinho lvvt+Pp

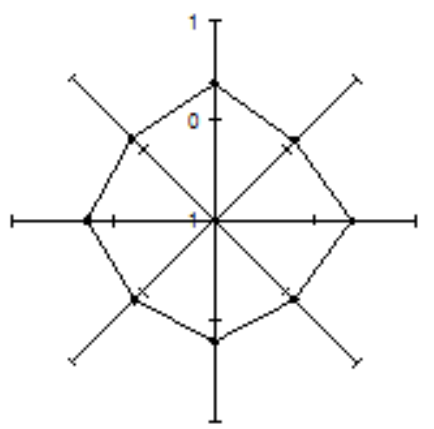

vinho lvvt+Pp+Pm

Figura 2 - Gráficos "star-plots" para os atributos de odor indesejado, acidez, taninos, adstringência, amargo, gosto indesejado, persistência e doçura, indicando o perfil de cada tratamento

No vinho $1 \mathrm{vvt}+\mathrm{Pp}$, o atributo mais intenso foi o odor indesejado. Além da intensidade de cor e gosto indesejado, os outros atributos obtiveram valores intermediários. Essas características indicam que o vinho inoculado com a levedura Pichia pijperi não agradou sensorialmente aos provadores.

O vinho $1 \mathrm{vvt}+\mathrm{Pm}$ apresentou três atributos de maior intensidade, segundo os provadores treinados: intensidade de sabor, intensidade de aroma frutado e intensidade de aroma. As menores notas estão relacionadas aos atributos odor indesejado e gosto indesejado, enquanto que os demais atributos ficaram próximos ou acima da média. Essas características indicam que o vinho inoculado com a levedura Candida valida agradou aos provadores, em particular, com relação ao aroma frutado.

$\mathrm{O}$ vinho 1vvt+Pp+Pm apresentou maior intensidade de cor do que os vinhos obtidos pelos demais tratamentos. Os atributos odor indesejado, acidez, taninos, adstringência, amargo, gosto indesejado, persistência e doçura apresentaram notas acima da média. As características acima apresentadas sugerem que o vinho inoculado com as leveduras Pichia pijperi e Candida valida tenha tido influência dessas duas leveduras não-Saccharomyces. Apesar desse vinho apresentar 
atributos desejáveis, os atributos odor indesejado e gosto indesejado tornam o vinho desagradável para os provadores.

A Análise de Componentes Principais (ACP) é uma representação gráfica, sugerindo apenas as tendências dos tratamentos e mostrando as relações existentes entre os tratamentos e os atributos que mais os caracterizam (de Faria e Yotsuyanagi, 2002). Todos os atributos avaliados possuem uma correlação entre si, gerada a partir da avaliação sensorial, em maior ou menor grau e de forma positiva ou negativa. Quando um atributo for intenso em um tratamento, o outro atributo também será intenso se os dois apresentarem correlações positivas. Já quando os atributos apresentam correlação negativa isso significa que quando um atributo for intenso em um vinho, o outro terá baixa intensidade (SHARMA, 1996).

A realização das correlações de Pearson permitiu identificar quais os atributos dos vinhos correlacionam-se significativamente, ao nível de 5\% de probabilidade. Considerou-se uma alta correlação entre as amostras quando os valores encontraram-se acima de 50\% (r > 10,501 ). Existe diferença significativa entre os atributos para as seguintes correlações positivas: atributo intensidade de aroma e intensidade de aroma frutado (49\%); a intensidade de sabor e de aroma (33\%); intensidade de aroma vegetal e persistência (33\%); intensidade de aroma vegetal e amargo (50\%); intensidade de aroma vegetal e adstringente (45\%); tanino e intensidade de sabor (44\%), persistência e intensidade de sabor (45\%); amargo e doçura (39\%); gosto indesejado e odor indesejado (62\%); adstringência e taninos (42\%); persistência e taninos (34\%); amargo e adstringência (56\%). As correlações negativas são encontradas entre os seguintes atributos: odor indesejado e intensidade de aroma frutado (48\%); a intensidade de cor e amargo (36\%); a intensidade de frutado e odor indesejado (48\%); persistência e acidez (38\%); qualidade geral e amargo (36\%); qualidade geral e persistência (61\%).

Os valores citados acima apresentaram baixo percentual de correlação. No entanto, os atributos gosto indesejado e odor indesejado estão altamente correlacionados positivamente (62\%), o sabor adstringente e sabor amargo também possuem uma alta correlação positiva (56\%) e o gosto indesejado e a persistência têm uma alta correlação negativa (61\%).

Por ser uma análise multivariada, a ACP permite a comparação da variância de cada um dos atributos em diversas dimensões espaciais. As dimensões - ou componentes - que justificarem a maior variabilidade dos dados serão as escolhidas para caracterizar as amostras, ou seja, aquelas que apresentarem os maiores valores de variância dos atributos (Sharma, 1996). Dos oito componentes gerados foram utilizados o Componente Principal 1 (PC1) e o Componente Principal 2 (PC 2) como sendo os dois componentes principais que justificaram a maior variabilidade total observada entre os atributos (64,58\%). A sobreposição dos gráficos dos atributos com os dos tratamentos permite a caracterização dos vinhos de acordo com suas posições nessas duas dimensões - Figura 3. 


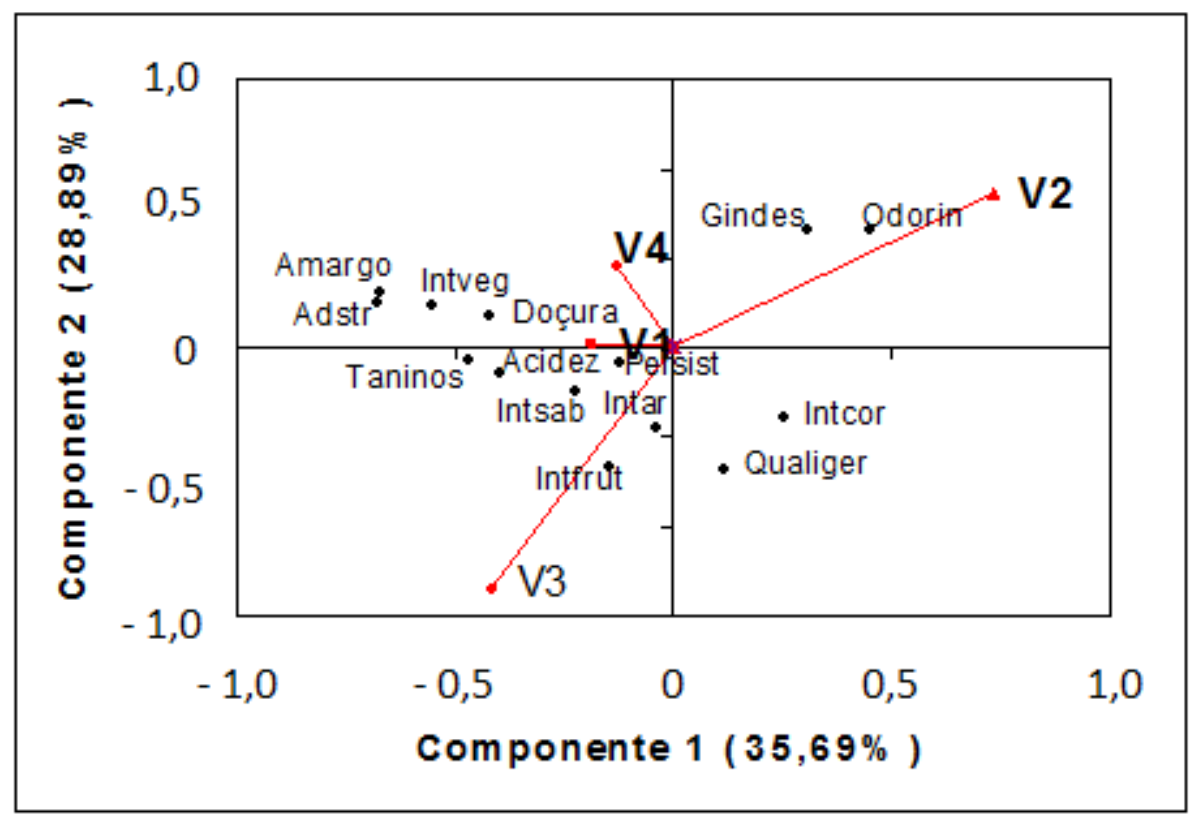

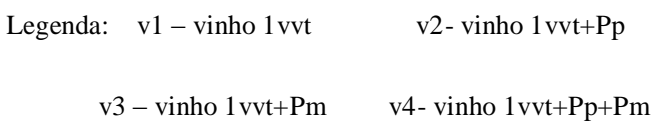

Atributos: Amargo (amargo); Doçura (doçura); Taninos (taninos); Adstr (adstringente); Intveg (intensidade de aroma vegetal); persist (persistência); Intsab (intensidade de sabor); Intar (intensidade de aroma) Intfrut (intensidade de aroma frutado); Intcor (intensidade de cor); Qualiger (qualidade geral); Gindes (gosto indesejado); Odorin (odor indesejado)

Figura 3 - Análise de componentes principais e correlação das amostras de vinho com os componentes

No gráfico da Análise de Componentes Principais, os vinhos similares ocupam regiões próximas no gráfico e são caracterizados pelos atributos que se apresentam mais próximos a eles. Pela posição dos tratamentos no gráfico nas dimensões 1 e 2 - Figura 3 - observa-se que os atributos gosto indesejado e odor indesejado, estão correlacionados positivamente com o vinho $1 \mathrm{vvt}+\mathrm{Pp}$. Este fato confirma, estatisticamente, a caracterização prévia do vinho no gráfico "star-plots" de que a aplicação da levedura Pichia pijperi torna o vinho desagradável.

Já os vinhos 1vvt e 1vvt+Pp+Pm, possuem valores de correlação com os componentes, próximo de zero, indicando que a variância apresentada por estes vinhos é dificilmente explicada simplesmente pelos dois componentes - Figura 3.

O vinho testemunha - 1vvt - possui características típicas do vinho Cabernet Sauvignon e o vinho inoculado com as leveduras não-Saccharomyces foi influenciado pelas duas leveduras, conforme a observação no gráfico “star-plots". Entretanto, os provadores treinados não conseguiram apresentar quais os atributos mais marcantes, que os diferenciam dos demais tratamentos.

No entanto o vinho $1 \mathrm{vvt}+\mathrm{Pm}$ está localizado no terceiro quadrante, onde se encontram os atributos intensidade frutado e intensidade de aroma próximos da reta de correlação, podendo este 
produto ser caracterizado pela tendência de aumento nos valores destas duas variáveis. O tratamento estatístico confirma a caracterização prévia do vinho inoculado com a levedura Candida valida no gráfico "star-plots" de que sua aplicação torna o vinho agradável sensorialmente, com relação à intensidade de aroma e intensidade de aroma frutado.

A Análise de Variância por ser um teste univariado permite a comparação de dois ou mais tratamentos - variáveis independentes - que gerem variáveis respostas de mesma escala e unidade (DE FARIA e YOTSUYANAGI, 2002). A ANOVA permite verificar quais atributos sensoriais dos quatorze avaliados são diferentes nos quatro vinhos elaborados. Na aplicação desta análise, apenas os atributos intensidade de cor e intensidade de aroma apresentaram diferença significativa dentre todos os atributos sensoriais avaliados, indicando que estes atributos foram os mais afetados pelos tratamentos ao nível de $5 \%$ de probabilidade $(\mathrm{p} \leq 0,05)$.

Em relação à qualidade geral, os vinhos não apresentaram diferença significativa ao nível de $5 \%$ de probabilidade, provavelmente, porque a variação de dois atributos não foi suficiente para afetar na avaliação do vinho como um todo.

A Análise de Variância diferenciou os vinhos pela intensidade de aroma, enquanto que a Análise de Componentes Principais demonstrou que o vinho inoculado com a Candida valida se caracterizou pela maior intensidade de aroma e intensidade de aroma frutado. A partir deste fato, conclui-se que a aplicação desta levedura melhorou a qualidade sensorial, aromatizante, do vinho Cabernet Sauvignon.

\section{Conclusão}

As duas linhagens Candida valida - Pm e Pichia pijperi - Pp apresentaram resultado positivo no teste de produção de sulfeto de hidrogênio. Entretanto, supõe-se que apenas a Pichia pijperi o tenha produzido em quantidades perceptíveis no vinho quando verificados os resultados das avaliações sensoriais. As análises químicas realizadas indicam que as leveduras Candida valida - Pm e Pichia pijiperi - Pp não prejudicam o desempenho da levedura de vinificação em processos fermentativos mistos.

$\mathrm{Na}$ avaliação sensorial as seguintes características foram observadas: os provadores não diferenciaram o vinho testemunha (1vvt) e o inoculado com as duas linhagens não-Saccharomyces $(1 \mathrm{vvt}+\mathrm{Pp}+\mathrm{Pm})$ dos demais vinhos; a inoculação da levedura Pichia pijperi tornou o vinho desagradável devido aos atributos gosto e odor indesejáveis; a inoculação da levedura Candida valida tornou o vinho mais agradável segundo os atributos intensidade de aroma e intensidade de aroma frutado. 
Os resultados das análises químicas e sensorial evidenciaram que a levedura Candida valida influenciou de forma benéfica na qualidade sensorial do vinho Cabernet Sauvignon. Isto indica a possibilidade de aplicação dessa levedura isolada de jaca (Arthocarpus hetherophyllus Lam) em vinificação com culturas mistas.

\title{
Agradecimentos
}

Ao CNPq pelo financiamento.

\begin{abstract}
Two non-Saccharomyces jackfruit's yeast strains - Pichia pijperi and Candida valida - were inoculated matched with Saccharomyces cerevisiae strain on four different winemakings. Chemical analyses showed that theses non-Saccharomyces yeast strains don't interfere on winemaking process. A trained panel sensory evaluated the wines on Sensory Descriptive Analysis and the data were treated by Principal Component Analysis and Variance Analysis. The wine inoculated with the yeast Pichia pijperi showed undesirable taste and smell attributes, while the attributes aroma and fruity intensity characterized the wine inoculated with Candida valida. The results pointed to the potential application of jackfruit's yeast Candida valida on winemaking culture starter.
\end{abstract}

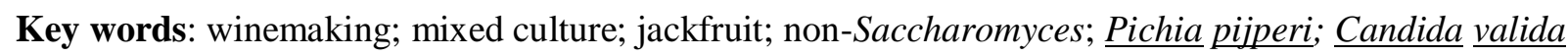

\section{Referências}

ABRANCHES, J.; VITAL, M. J. S.; STARMER, W. T.; MENDONÇA-HAGLER, L. C.; HAGLER, A. N. The yeast community and mycocin producers of guava fruit in Rio de Janeiro, Brasil. Mycologia, v. 92, n. 10, p. 16-22, 2000. http://dx.doi.org/10.2307/3761445

AIZAC, T.; DELTEIL, D.; VEZINHET, F. Etude écologique de souches de levures contaminantes. Application de la technique d’identification par caryotypes. Revue Française des Oenologues, v. 130, p. 6-20, 1991.

AMERINE, M. A.; ROESSLER, E. B. Wines - their sensory evaluation. W.H. San Francisco: Freeman and Company, 1983. 432p.

AMERINE, M. A.; CRUESS, W. V. The technology of wine making. The AVI Publishing Company, Inc., Westport, Connecticut, 1960.

BOUlTON, R. B.; SINGLETON, V. L.; BISSON, L. F.; KUNKEE, R. E . Principles and practices of winemaking. New York: Chapman e Hall, 1996.

CHIAPPINI, C. C. J. Caracterização química e sensorial do aroma produzido por leveduras isoladas de jaca (Artocarpus heterophyllus Lam) e aplicação em mosto de uva. Rio de Janeiro: Instituto de Química, UFRJ, 2004. Tese de Doutorado.

COMBINA, M.; ELÍA, A.; MERCADO, L.; CATANIA, C.; GANGA, A.; MARTINEZ, C. Dynamics of indigenous yeast populations during spontaneous fermentation of wine from Mendonza, Argentina. International Journal of Food Microbiology, v. 99, p. 237-243, 2005. http://dx.doi.org/10.1016/j.ijfoodmicro.2004.08.017

CONSTANTI, M.; POBLET, M.; AROLA, L. MAS, A. e GUILLAMON, M. Analysis of yeast populations during alcoholic fermentation in a newly established winery. American Journal of Enology and Viticulture, v. 48, p. 339344, 1997.

CUINIER, P.; BOUIX, N.; LEVAN, J. Méthode d'étude de l'origine des levures en oenologie: identification des espéces et diferentation des clones. Vignes Vins, v. 302, n. 37, 1981. 
FARIA, E. V.;YOTSUYANAGI,K. Técnicas de análise sensorial. Campinas: ITAL/ LAFISE, 2002. 116p.

GAFNER, J.; HOFFMANN-BOLLER, P.; PORRET, N.; PULVER, D. Winemaking microbiology: advances in research and their impact on winemaking practices. Wine biotechnology, p. 258-260, 2000.

GROSSMANN, M.; LINSENMEYER, H.; MUNO; H.; RAPP, A. Use of oligo-strain yeast cultures to increase complexity of wine aroma. Viticulture and Enological Sciences, v. 51, p. 175-179, 1996.

HEARD, G. M.; FLEET, G. H. The effects of temperature and $\mathrm{pH}$ on the growth of yeast species during the fermentation of grape juice. Journal Applied Bacteriology, v. 65, p. 23-28, 1988. http://dx.doi.org/10.1111/j.13652672.1988.tb04312.x

JOLLY, N. P.; AUGUSTYN, O. P. H.;PRETORIUS, I. S.The occurence of non-Saccharomyces yeast strains in four vineyards and grape musts in four production regions of Western Cape, South Africa. South Africa Journal of Enologie and Viticulture, v. 24, n. 2, p. 35-42, 2003a.

JOLLY, N. P.; AUGUSTYN, O. P. H.; PRETORIUS, I. S. The effect of non-Saccharomyces yeasts on fermentation and wine quality. South Africa Journal of Enologie and Viticulture, v. 24, n. 2, p. 55-62, 2003 b.

JOLlY, N. P.; AUGUSTYN, O. P. H.; PRETORIUS, I. S. The use of Candida pulcherrima in combination with Saccharomyces cerevisiae for the production of Chenin blanc wine. South Africa Journal of Enologie and Viticulture, v. 24, n. 2, p. 63-69, 2003c.

LE ROUX, G.; ESCHENBRUCH, R.; DE BRUIN, S. I. The microbiology of South African wine-making. Part.VIII. The microflora of healthy and Botrytis cinerea infected grapes. Phytophylactica,v. 5, p. 51-54,1973.

LONVAUD-FUNEL, A. Microorganisms on winemaking. Cerevisiae: Belgican Journal of Brewing Biotechnology, 21: 55-58, 1996.

MORAIS, P. B.; ROSA, C. A.; MEYER, S. A.; MENDONÇA-HAGLER, L. C.; HAGLER, N. Candida amapae, a new amino acid-requiring yeast from Amazonian fruit Parahancornia amapa. Journal of Industrial Microbiology, v. 14, n. 1-6, p. 531-535, 1995a.

MORAIS, P. B.; MARTINS, M. B.; KLACZKO, L. B.; MENDONÇA-HAGLER, L. C. ; HAGLER, N. Yeast succession in the Amazon fruit Parahancornia amapa as resource partitioning among Drosophila spp. Applied and Environmental Microbiology, v. 61, n. 12, p. 4251-4257, 1995b.

PHAFF, H.J.; STAMER, W.T. Yeasts associated with plants, insects and soil. In: Rose, A.H. e Harrison, J. The yeasts, v.1. London: London Press, 1987. p. 123-180.

PEYNAUD, E; DOMERCQ, S. A review o microbiological problems in wine-making in France. American Journal of Enology and Viticulture, v. 10, p. 69-77, 1959.

RANKINE, B. C. Influence of yeast strain and malo-lactic diferentation on composition and quality o table wines. American Journal of Enology and Viticulture, v. 23, p. 152-158, 1972.

RIBÉREAU-GAYON, J.; PEYNAUD, E.; RIBÉREAU-GAYON, P.;SUDRAU, P. Traité d’onologie: sciences et techniques du vin. Tome 1. Paris: Dunod, 1976. 719 pp.

RIBÉREAU-GAYON, J.; PEYNAUD, E.; RIBÉREAU-GAYON, P.;SUDRAU. ,P. Traité doenologie: sciences et techniques du vin. Tome 2. Paris: Dunod, 1976. 719 pp.

RIBÉREAU-GAYON, J.; GLOIRES, Y.; MAUJEAN, A.; DUBORDIERr, D. Phenolic compounds. In: RIBERÉAUGAYON, P. GLOIRES, Y.; MAUJEAN, A.; DUBORDIER, D. Handbook of enology v2. The chemistry of wine stabilization and treatments. New York: John Wiley e Sons Ltd., 2002. 404pp.

RIBÉREAU-GAYON, J.; PEYNAUD, E. Analyses et Controle des Vins. 2ed. Paris: Librairie Polytechnique Ch. Béranger, 1958.

ROMANO, P.; FIORE, C.; PARAGGIO, M.; CARUSO, M.; CAPECE, A. Functional of yeast species and strains in wine flavour. International Journal of Food Microbiology, v. 86, p. 169-180, 2003. http://dx.doi.org/10.1016/S01681605(03)00290-3 
ROSINI, G. Assessment of dominance of added yeast in wine fermentation and origin of Saccharomyces cerevisiae in wine-making. Journal of General Applied Microbiology, v. 30, p. 249-256, 1984. http://dx.doi.org/10.2323/jgam.30.249

ROSA, C. A.; MORASI, P. B.; SANTOS, S. R.; NETO, P. R. P.; MENDONÇA-HAGLER, L. C.; HAGLER, A. N. Yeasts communitie associated with different plant resources in sandy coastal plains of southeastern Brazil. Mycological Research, v. 9999, p. 1047-1054, 1995. http://dx.doi.org/10.1016/S0953-7562(09)80771-5

SANTOS, E. A.; OLIVEIRA, E. A.; MENDONÇA-HAGLER, L. C. e HAGLER, A. N. Yeasts associated with flowers and fruits from a semi-arid region of Northeastern Brazil. Revista de Microbiologia, v. 27, n. 1, p. 33-40, 1996.

SLINKARD, K.; SINGLETON, V. L. Total Phenol Analysis: automation and comparison with manual methods. American Journal of Enology and Viticulture, v. 28, p. 49-55, 1977.

VAN ZYL, J. A.; DU PLESSIS, L. The microbiology of South African winemaking. Part I. The yeasts occurring in vineyard, must and wines. South Africa Journal of Agricultural Science, v 4, p. 393-403, 1961.

VINE, R. P.; HARKNESS, E. M.; BROWING, T.; WAGNER, C. Winemaking: from grape growing to marketplace. New York: Chapman e Hall Enology Library, 1997. p.439.

WATERHOUSE, A. Folin-Ciocalteau micro method for total phenol in wine, 2005. Disponível em: <http://waterhouse.ucdavis.edu/phenol/folinmicro.htm >

Submetido em 10 jan. 2011; Revisão submetida pelos autores em 09 out 2011, Aceito para publicação em 14 out. 2011. 